\section{Lack of directional specificity in tilt aftereffect induced with moving contours*}

\author{
RAY OVER $†$ and JACK BROERSE \\ University of Queensland, St. Lucia 4067, Australia
}

Eight Ss were required to set a grating to vertical following exposure to a tilted grating. Aftereffect was measured with both gratings stationary, with one grating stationary and the other moving, or with both gratings moving either in the same or in opposite directions. The mean tilt aftereffect did not vary among these conditions. The results are discussed with reference to explanations of visual tilt aftereffect that have been offered in terms of selective suppression of spatially tuned feature detectors.

A vertical line or grating looks slightly tilted when viewed immediately following inspection of tilted lines. Several recent accounts of this visual tilt aftereffect (e.g., Coltheart, 1971; Over, 1971) are based on the assumption that the tilt of a line is uniquely represented in the visual cortex by a specific distribution of excitation within a set of cells differentially tuned to contour orientation. Perceptual distortions found when the vertical line is viewed following inspection of tilted lines are attributed to proactive inhibitory interaction between neural analyzers that signal the orientation of each line. The claim is that cells that were excited during exposure to tilted lines are temporarily suppressed when the vertical line is displayed, whereas the response of cells tuned to the postinspection but not to the inspection orientation is unaffected. Accordingly, the discharge pattern generated by the postinspection stimulus is that normally signaled by a line at a slightly different orientation. Over (1971) has discussed neurophysiological and psychophysical studies that provide information about feature analysis in the visual system relevent to this explanation of visual tilt aftereffect.

The above analysis implies that tilt aftereffects can be induced only when the spatial properties of the inspection and postinspection stimuli are represented within the same set of neural units, No aftereffect would result, for example, if all cells responsive to the postinspection stimulus were tuned so that they were not involved in signaling the spatial properties of the inspection stimulus.

*This research was supported by an award to the first author from the Australian Research Grants Committee. Thanks are given to Ann-Marie Parker for her assistance in data collection and analysis.

tRequests for reprints should be sent to Ray Over, Dept. of Psychology, University of Queensland, St. Lucia 4067, Australia.
Microelectrode studies have demonstrated that a variety of spatial-analyzing operations are performed in the cat visual cortex. Some (but not all) cells are sensitive to the direction of image motion and are also excited optimally at a specific stimulus velocity (Pettigrew, Nikara, \& Bishop, 1968). Units optimally responsive to low rates $(4 \mathrm{deg} / \mathrm{sec}$ or less) predominate. These cells do not, however, function exclusively as velocity detectors, as their responses can be modified by variation in the direction of image motion (Pettigrew, Nikara, \& Bishop, 1968) or in the periodicity of the stimulating contours (Campbell, Cooper, \& Enroth-Cugell, 1969).

If neural analyzers have compound spatial selectivity, it may be possible to induce tilt aftereffects only if the inspection and postinspection stimuli differ in orientation but are similar in other spatial properties. Psychophysical experiments employing masking paradigms have provided indirect evidence that analyzers with compound spatial selectivity exist in the human visual system. Exposure to one grating impairs detection of another grating to the extent that the two gratings are similar in spatial frequency, but periodicity masking is limited by angular differences between the two gratings (Blakemore \& Nachmias, 1971) as well as by differences in the velocity of motion of inducing and test gratings (Pantle, 1970). In addition, the periodicity aftereffect is critically affected by the tilt relationship between the inspection and test contours (Blakemore \& Nachmias, 1971).

The present experiment examined the selectivity of the visual tilt aftereffect to the direction of image motion. The inspection and postinspection stimuli were gratings, which were either stationary or moving in a specific direction. The S's task was to set the test grating to the apparent vertical immediately after inspection of a grating tilted $0,15,30$, or $45 \mathrm{deg}$ from vertical. Measures were obtained with both gratings stationary, with one grating stationary and the other moving, and with both gratings moving. Under the latter condition, the gratings moved in either the same or opposite directions. It was asked, within the context of the model of aftereffects considered earlier, whether the tilt aftereffect could be induced only when the inspection and postinspection gratings moved in the same direction.

\section{METHOD}

Square-wave grating patterns were formed on an oscilloscope (Advance Model 2000S) by a method similar to the technique described by Campbell \& Green (1965). The phosphor had broad spectral wavelength emission, and its intensity could be modulated linearly with applied voltage. The vertical $Y$ axis was driven by an internal amplifier from an external oscillator delivering a $100-\mathrm{kHz}$ sine wave, the horizontal $\mathrm{X}$ axis was driven by the oscilloscope's internal time-base circuit at $2,000 \mathrm{~Hz}$, and the electron beam was modulated by a variable square-wave generator. By applying a synchronization pulse from this generator to the external trigger input of the time-base circuit, a grating could be locked in a stationary position. When the synchronization pulse was disconnected, the grating moved from left to right or from right to left at a velocity dependent on the harmonic relationship between the square-wave and the fundamental $100-\mathrm{kHz}$ sine wave.

Separate oscilloscopes displayed the gratings used as inspection and test stimuli, and a half-silvered mirror arrangement was employed so that the two gratings could be presented successively to the same part of the visual field. A shutter system was used to occlude one grating while the other grating was being displayed. The two gratings were circular, subtending $3 \mathrm{deg} 20 \mathrm{~min}$. The spatial frequency was maintained at 3 cycles/deg, and when a grating was moving, its velocity was $2 \mathrm{deg} / \mathrm{sec}$. The image orientation of each grating could be varied by rotation of a Dove prism located between the viewing aperture and the half-silvered mirror. When making postinspection judgments, the $S$ was able to rotate the prism by hand-operated controls until the grating appeared vertical, and $\mathrm{E}$ recorded the setting from a protractor reading to the nearest $1 / 2 \mathrm{deg}$.

The eight Ss were undergraduate and postgraduate students. Each $S$ was tested under five conditions: inspection stationary/test stationary, inspection stationary/test moving left to right, inspection moving left to 
Table 1

Aftereffect in Degrees Across the Five Testing Conditions

\begin{tabular}{ccccccc}
\hline \multicolumn{2}{c}{ Motion Properties of Gratings } & & \multicolumn{3}{c}{ Orientation of Inspection Grating } \\
\cline { 5 - 7 } Inspect & Test & & 0 & 15 & 30 & 45 \\
\hline Stat & Stat & M $\rightarrow$ & .09 & $1.34^{*}$ & $1.06^{*}$ & -.03 \\
Stat & Stat & .66 & $1.22 \dagger$ & $1.44^{*}$ & .38 \\
$M \rightarrow$ & $M \rightarrow$ & -.19 & $1.19 \dagger$ & .25 & $1.06 \dagger$ \\
$M \rightarrow$ & $M \rightarrow$ & .41 & $1.25 *$ & .75 & -.19 \\
$M \leftarrow$ & & .19 & $1.91 \dagger$ & $.97^{*}$ & .56 \\
\hline
\end{tabular}

$* p<.05 \quad t p<.01$

right/test stationary, inspection moving left to right/test moving left to right, inspection moving right to left/test moving left to right. In each case, four measures of aftereffect were obtained with the inspection grating tilted $0,15,30$, and $45 \mathrm{deg}$ from vertical. Values were obtained under a given condition and at a specific angle in the following manner. Four preinspection settings, two each from a starting position $10 \mathrm{deg}$ to the left and $10 \mathrm{deg}$ to the right of vertical, were made initially. Four postinspection settings, two from each starting position, were then obtained. Each was preceded by a 30-sec inspection period. To control for differential decay in aftereffect due to judgment time, the $S$ s were required to complete all settings within $4 \mathrm{sec}$ and were given a count of elapsed time by E. The sequency in which the conditions were tested was varied across Ss by a single Latin square and three randomly chosen orders. Testing was conducted in a darkened room, and the gratings were viewed against a homogeneously dark background. Viewing was monocular with the right eye, and fixation was not required.

\section{RESULTS}

Aftereffects were measured in terms of differences between preinspection and postinspection settings made from a common starting position. Table 1 shows the mean aftereffect obtained under the five testing conditions and for the four angles of tilt of the inspection grating. The aftereffect has been scored + if the postinspection setting was displaced from the preinspection value in the direction of tilt of the inspection grating and - if the displacement was in the opposite direction. When the inspection grating was vertical, + was scored if the test figure was displaced in the clockwise direction and - if counterclockwise displacement occurred. Table 1 also indicates whether the mean aftereffect in each case differs significantly from zero.

An analysis of variance was performed on measures obtained at inspection angles of 15,30 , and
45 deg. In accord with previous experiments (see Over, 1971), mean aftereffect varied significantly as a function of the orientation of the inspection grating, $F(2,14)=7.39$, $\mathrm{p}<.01$. However, the motion characteristics of the inspection and test gratings had no influence over the magnitude of the tilt aftereffect, $F(4,28)=.77, p>.05$, and the interactiobetween orientation and motion characteristics was insignificant, $F(8,56)=2.26, p>.05$. Multiple comparisons based on Duncan's test revealed no differences in peak aftereffect between the five testing conditions.

\section{DISCUSSION}

The present results clearly indicate that the visual tilt aftereffect is selective to contour orientation relationships but not to direction of image motion. It was possible to induce an aftereffect using either stationary or moving test gratings, and the aftereffect was not diminished when $S$ was required to judge the orientation of contours moving left to right after inspection of tilted lines moving right to left. If the model of tilt aftereffects considered earlier is to be maintained, the present results must be interpreted as indicating that neural units are tuned to contour orientation without regard to the motion properties of the stimulus. If this were the case, the visual tilt aftereffect induced with moving contours should lack velocity selectivity and not only directional

This lack of directional selectivity in the orientation aftereffect found with moving contours does not necessarily invalidate the explanation of the tilt aftereffect proposed earlier. Directional selectivity would be expected only if motion detectors could each be excited by image motion in a single direction. Microelectrode studies (e.g., Campbell, Cleland, Cooper, \& Enroth-Cugell, 1968; Pettigrew, Nikara, \& Bishop, 1968) have, in fact, shown that a large proportion of motion-sensitive cells in the cat visual cortex have two selectivity. preferred directions of movement $180 \mathrm{deg}$ apart. Masking studies (e.g., Pantle \& Sekuler, 1969) indicate that detection of a moving target sitmulus is impaired as a function of the direction and velocity of the preceding adaptation stimulus. Nevertheless, detection of downward motion is impaired by exposure to upward motion relative to measures obtained after exposure to a stationary grating. Such data suggest that the human visual system contains both directional and nondirectional motion detectors.

In these terms, the explanation of tilt aftereffect outlined earlier can at best predict that the aftereffect will be smaller when the inspection and test gratings move in opposite directions than when they move in the same direction. However, the present data fail to provide evidence that the tilt aftereffect is selective in any way to the direction of image motion. It has similarly been shown (Campbell \& Maffei, 1971) that the tilt aftereffect is unaffected by the spatial periodicity relationship between inspection and test gratings, even though masking data (Blakemore \& Nachmias, 1971) suggest that at least some orientation detectors in human vision are also tuned to spatial frequency.

\section{REFERENCES}

BLAKEMORE, C., \& NACHMIAS, J. The orientation specificity of two visual aftereffects. Journal of Physiology, 1971, 213, 157-174.

CAMPBELL, F. W., CLELAND, B. G., COOPER, G. F., \& ENROTH-CUGELL, C. The angular selectivity of visual cortical cells to moving stimuli. Journal of Physiology, 1968, 198, 237-250.

CAMPBELL, F. W., COOPER, G. F., \& ENROTH-CUGELL, C. The spatial selectivity of the visual cells of the cat. Journal of Physiology, 1969, 203, 223-235.

CAMPBELL, F. W., \& GREEN, D. G. Optical and retinal factors affecting visual resolution. Journal of Physiology, 1965, $181,576-593$.

CAMPBELL, F, W, \& MAFFEI, L. The tilt after-effect: A fresh look. Vision Research, 1971, 11, 833-840.

COLTHEART, $M$. Visual feature-analyzers and aftereffects of tilt and curvature. Psychological Review, 1971, 78, 114-121.

OVER, R. Comparison of normalization theory and neural enhancement explanation of negative aftereffects. Psychological Bulletin, 1971, 75, 225-243.

PANTLE, A. Adaptation to pattern spatial frequency: Effects on visual movement sensitivity in humans. Journal of the Optical Society of America, 1970, 60, 1120-1124.

PANTLE, A., \& SEKULER, R. Contrast response of human visual mechanisms sensitive to orientation and direction of motion. Vision Research, 1969, 9, 397-406.

PETTIGREW, I. D, NIKARA, T. \& BISHOP, P O Responses to moving slits by single units in cat striate cortex. Experimental Brain Research, 1968, 6, 373-390. 